\title{
Factors influencing the integration of a palliative approach in intensive care units: a systematic mixed-methods review
}

\author{
Hanan Hamdan Alshehri ${ }^{1,2}$, Sepideh Olausson ${ }^{1}$, Joakim Öhlén ${ }^{3,4}$ and Axel Wolf ${ }^{1,5^{*}}$ (D)
}

\begin{abstract}
Background: While a palliative approach is generally perceived to be an integral part of the intensive care unit (ICU), the provision of palliative care in this setting is challenging. This review aims to identify factors (barriers and facilitators) influencing a palliative approach in intensive care settings, as perceived by health care professionals.

Method: A systematic mixed-methods review was conducted. Multiple electronic databases were used, and the following search terms were utilized: implementation, palliative care, and intensive care unit. In total, 1843 articles were screened, of which 24 met the research inclusion/exclusion criteria. A thematic synthesis method was used for both qualitative and quantitative studies.

Results: Four key prerequisite factors were identified: (a) organizational structure in facilitating policies, unappropriated resources, multi-disciplinary team involvement, and knowledge and skills; (b) work environment, including physical and psychosocial factors; (c) interpersonal factors/barriers, including family and patients' involvement in communication and participation; and (d) decision-making, e.g., decision and transition, goal conflict, multidisciplinary team communication, and prognostication.

Conclusion: Factors hindering the integration of a palliative approach in an intensive care context constitute a complex interplay among organizational structure, the care environment and clinicians' perceptions and attitudes. While patient and family involvement was identified as an important facilitator of palliative care, it was also recognized as a barrier for clinicians due to challenges in shared goal setting and communication.
\end{abstract}

Keywords: Palliative care, Critical care, Implementation science, Intensive care units, Review

\section{Background}

Intensive care units (ICUs) are specialized hospital wards where seriously ill patients, often with life-threatening conditions, are treated by specialized care professionals and receive intensive care [1], with the essential aim of providing life-saving and life-sustaining care [2, 3]. While mortality rates have drastically improved in recent

\footnotetext{
*Correspondence: axel.wolf@gu.se

'Institute of Health and Care Sciences, Sahlgrenska Academy, University of Gothenburg, Gothenburg, Sweden

${ }^{5}$ Department of Anaesthesiology and Intensive Care Medicine, Region Västra Götaland, Sahlgrenska University Hospital/Östra, Gothenburg, Sweden Full list of author information is available at the end of the article
}

decades [4], a substantial proportion of patients receive intensive care who will either not survive or who will be discharged for end-of-life care, often close to death [5]. This highlights the relevance of integrating a palliative approach into the care process in intensive care settings. Thus, during recent decades, the point of departure of critically ill patients within the ICU has changed, shifting towards more chronically ill patients who deteriorate in their chronic condition. The majority of patients in the ICU are elderly, representing a vulnerable population with a history of chronic and often comorbid diseases such as heart failure or COPD. The COVID-19 
pandemic shows this very clearly, as many elderly patients with chronic diseases are admitted to the ICU due to respiratory failure [6, 7]. Clearly, the COVID-19 pandemic necessitates the inclusion of responses with a palliative approach, such as active symptom relief, communication and recognition of dying. When chronic conditions lead to deterioration and occur among older adults, the question of the integration of a palliative approach to patient comfort and end-of-life care intensifies.

In general, a palliative approach aims to relieve suffering for patients with life-limiting conditions and for those who are dying and to manage symptoms, increase the level of care comfort and provide support to family members $[8,9]$. Despite increasing awareness of integrating a palliative care approach in the ICU, there are challenges, given the somewhat contradictory aims of intensive care and palliative care, i.e., in providing lifesaving treatments vs treating dying as a normal process. However, studies have shown the benefits of integrating palliative care into intensive care [10-13], for example, in relieving distress for patients and their families during end-of-life care. Nevertheless, a review by Kahveci [14] on attitudes and beliefs pertaining to integrating a palliative approach in the ICU shows that there are many challenges, such as sociocultural factors, legal regulations and a lack of awareness of a palliative approach. The integration of a palliative approach has been described as challenged by a lack of resources for symptom management and cultural and societal values and beliefs about death and dying [15]; moreover, structural barriers (for example, limited specialties and resources) appear especially difficult to change [16].

To date, no mixed-methods review has been conducted that integrates both quantitative and qualitative evidence to frame a broader picture of factors that influence the adoption of a palliative care approach in the ICU. Hence, there is a need to synthesize the findings of quantitative and qualitative research studies on the factors (facilitators and barriers) influencing a palliative approach in the ICU from the perspective of allied health professionals. In particular, there is a need to increase knowledge of contextual factors (attitudes, perceptions and structural/organizational) influencing this integration of care perspectives, which could be considered to be exclusively related to each other.

\section{Aim}

To identify factors influencing a palliative approach in intensive care units, as perceived by health professionals.

\section{Methods}

A mixed-methods systematic review was undertaken with the aim of identifying, assessing, analysing and synthesising the current research findings [17]. The first step in the process was to perform a review protocol (registered in the international prospective register of systematic reviews (PROSPERO) (CRD42018099786). Second, systematic literature searches were conducted, and relevant literature was selected. Third, we performed quality assessments of the included articles [18]. Finally, we analysed and synthesised the articles' findings, taking the assessed quality into consideration.

\section{Eligibility criteria and review selection}

A search guide was developed based on the research concepts and questions within the inclusion and exclusion criteria. The authors used a PEO framework (population, exposure, outcome) [17] and focused on the following:

Population - allied health care professionals in the ICU;

Exposure - integration or implementation of a

palliative approach;

Outcome - factors (facilitators and barriers)

influencing a palliative approach; and.

Context - ICU.

Two expert medical librarians supported our search process.

The inclusion criteria were as follows:

(1) Studies focusing on factors influencing the integration of a palliative approach for adult patients admitted to the ICU;

(2) Studies highlighting health care professionals' experiences or perceptions of the integration or implementation of palliative care in ICUs;

(3) Studies written in the English language; and.

(4) Peer-reviewed studies published between January 2007 and January 2018.

Our exclusion criteria were as follows:

(1) Non-empirical studies (e.g., editorials, brief reports);

(2) Studies in paediatric and neonatal intensive care;

(3) Studies reporting the frequency of palliative care in the ICU and the effect of palliative care on the mortality rate or length of stay in the ICU; and

(4) Studies regarding palliative care policy.

\section{Literature search strategy}

In this review, we have chosen to use broad concepts and surrogate words and associated definitions. We used the search terms targeting the integration of a palliative approach, such as implementation, palliative care, and intensive care units, as well as synonyms for these terms (see Additional file 1). The university librarian at the 
University of Gothenburg performed an electronic database search of the following databases: AMED, PubMed, EMBASE, PsycInfo, Sociological Abstracts, Web of Science, Scopus and Cinahl. In total, 1843 citations were identified (AMED $n=21$, PubMed $n=507$, EMBASE $n=$ 376, PsycInfo $n=46$, Sociological Abstracts $n=3$, Web of Science $n=160$, Scopus $n=495$, and Cinahl $n=235$ ).

\section{Selection of articles}

In total, 1843 articles were identified in the initial search, of which 462 were duplicates and were thus deleted before we imported the remaining 1407 articles into an web based systematic reviews software for blind screening (Rayyan QCRI, developed by Qatar Computing Research Institute) (see Fig. 1). Two authors (HH, AW) performed blind screening of the articles in Rayyan following the inclusion and exclusion criteria (above). In the first round, the article titles and abstracts were screened (in the online tool Rayyan). In the second round, eligibility was assessed based on the blind reading of 158 full-text articles by two authors (HH, AW). The authors summarized and documented the reasons for inclusion and exclusion. In the third round, which was unblinded, two authors ( $\mathrm{HH}, \mathrm{AW})$ compared and discussed the relevant articles, and if there was disagreement, consensus for final inclusion was achieved by consulting all members of the research team. As a result, 24 articles were ultimately included in this systematic review (see Fig. 1).

\section{Quality assessment}

Depending on the type of study, the quality assessment was carried out as follows: nine qualitative studies and one quantitative study with an RCT design were evaluated using the Critical Appraisal Skills Program (CASP). The remaining quantitative studies $(n=8)$ were evaluated using the Best Evidence topics (Best BETs) critical appraisal checklists for survey study design. The MixedMethods Appraisal Tool (MMAT) was used to evaluate the studies using a mixed-methods approach $(n=6)$. The researchers evaluated each of the studies and developed scores for all types of tools. For the quality assessment summary (see Additional file 2).

\section{Analysis and synthesis}

A thematic synthesis approach was selected for the study. Thematic analysis has been used in mixedmethods systematic reviews that address questions such as identifying barriers or facilitators from evidence and

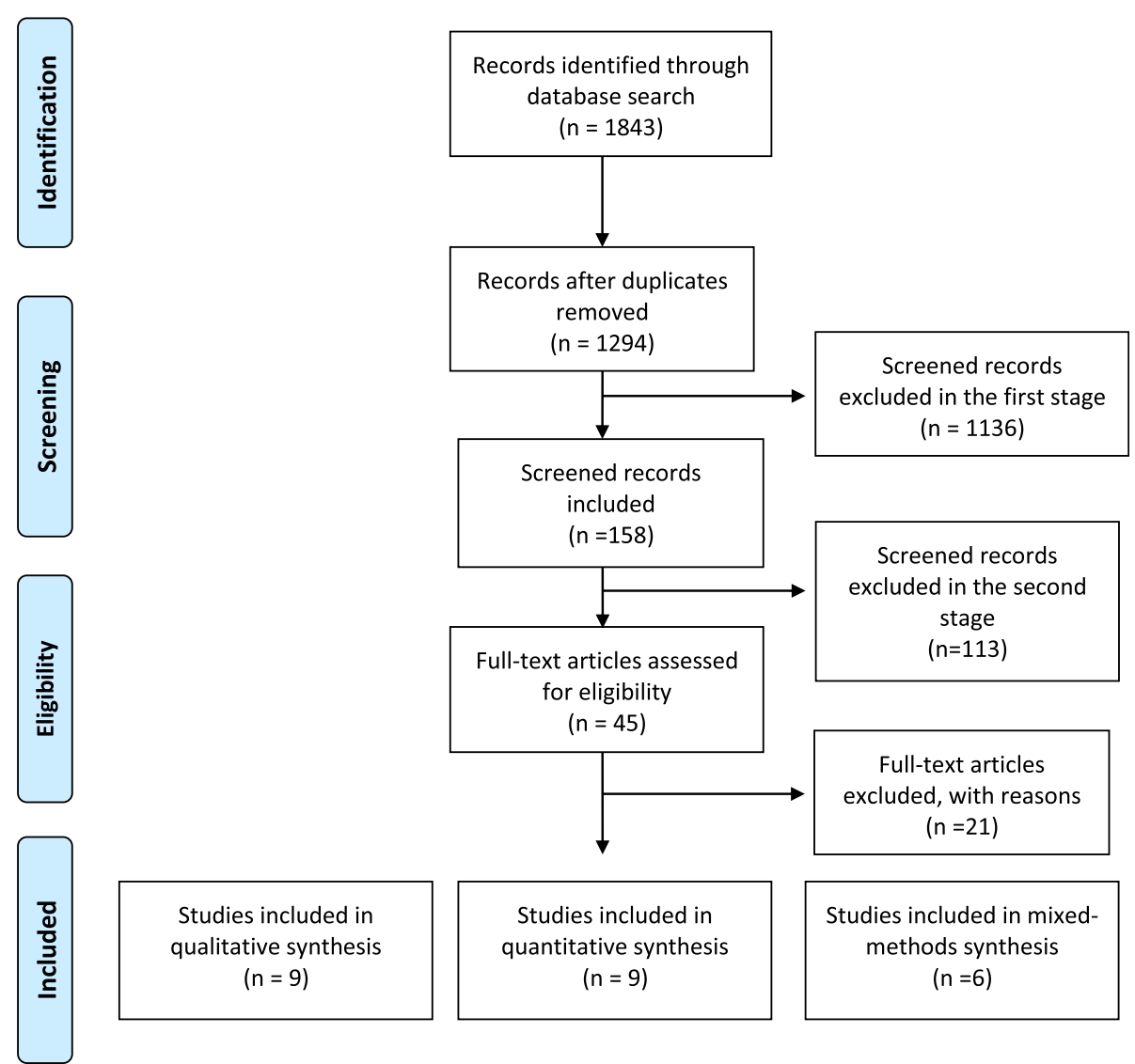

Fig. 1 PRISMA flowchart 
identifying patterns within the findings [17]. While thematic synthesis is generally used for the synthesis of qualitative studies, it may also be useful when there is heterogeneity in outcome variables and measurement in quantitative studies. Before the beginning of the analysis stages, the first author $(\mathrm{HH})$ read each included article several times, data were extracted, and quality assessments were performed for quantitative and qualitative analyses separately. A three-stage thematic analysis process was undertaken [18]. In the first stage, the first author $(\mathrm{HH})$ focused on factors influencing a palliative approach in the ICU based on results from quantitative data. In the second stage, we focused on factors influencing a palliative approach in the ICU based on results from qualitative data. In the third stage, two authors $(\mathrm{HH}, \mathrm{AW})$ integrated the analyses using thematic synthesis with a focus on factors influencing a palliative approach in the ICU based on results from both quantitative and qualitative data. They also repeatedly checked the analysis against the articles and started to thematize the findings into influencing factors. Inconsistencies in preliminary analysis were discussed, and the other authors were consulted (SO, JÖ). In this stage, we sought to distinguish the influencing factors into overarching analytical categories (type of factors) and more descriptive categories (specific factors). The similarities and differences between the findings were highlighted and grouped to finalise the development of descriptive categories (factors) and analytical categories (type of factors). All authors were involved in reviewing and checking the accuracy of the final findings.

\section{Results}

Study characteristics and quality assessment summary: Fig. 1 shows a standard flow chart reporting the results of the bibliographic search and screening (it follows the guidance of the Preferred Reporting Items of Systematic Reviews and Meta-Analyses (PRISMA). In total, 24 studies were eligible for the review: nine qualitative, nine quantitative and six mixed-methods studies. In total, the articles reported studies with 2545 participating allied health professionals. Studies were conducted in ten countries worldwide, with most studies conducted in the United States of America $(n=11)$, the United Kingdom $(n=5)$, the Netherlands (2), Germany (2), Australia (1), Canada (1), Japan (1) and Brazil (1). A summary of all included articles and their quality assessments are shown in (see Tables 1, 2, and 3). In total, eight articles were assessed as having either moderate-to-high or high quality, eight articles were assessed as having moderate quality, four articles were assessed as having low-to-moderate quality, and four articles were assessed as having low quality (reference [33, 34, 38, 40]) (see additional file 2).

\section{Influencing factors (facilities and barriers)}

Four types of influencing factors were identified: (1) organizational structures, (2) working environment, (3) patient and family involvement, and (4) palliative care decision-making. We present the summary of these types of influencing factors below and specific factors with related facilitators and barriers in Table 4 Below, each of the four influencing factors are presented with specific facilitating and hindering factors.

\section{Organizational structures}

Several studies highlighted the lack of protocols and policies for integrating a palliative approach in the ICU [22, 23, 34, 37]. Nevertheless, several studies have also revealed that physicians and nurses tend to be resistant to and unaware of the guidelines for a palliative approach in the ICU [26, 37, 38]. The number of staff, the standardization of the staffing ratio [27], and the time spent by staff with patients were examples of factors influencing the integration of a palliative approach in the ICU $[19,34]$. There was a lack of organizational support, not in the least for junior nurses, who reported a lack of mentoring and support.

Several studies indicated that poor education and knowledge about a palliative approach created barriers and were due to inadequate education and knowledge among nurses working in the ICU [21,38]. One study reported that nurses gained their palliative care experience through trial and error [19]. Other barriers identified were insufficient information [32], a lack of awareness of the complexity of and the communication required for a palliative approach in the ICU, inadequate training in palliative care decision making [29,32], and a lack of specialized palliative care teams, which was an obstacle to integrating a palliative approach for patients in the ICU $[37,40]$.

A palliative approach was positively enhanced by following standardized tools for dialogue [38], bereavement programmes, and adherence to the appropriate policies and procedures [26]. This approach was also enhanced by the introduction of team meetings [36], collaboration with other specialties [20, 39], the involvement of families into the multidisciplinary discussion [22, 34]. Palliative care training programmes for critical care professionals, or peer-to-peer support programmes, improved the integration of a palliative approach in the ICU [36], as well as specialized palliative care teams' participation and mentoring within the ICU teams [25].

\section{Working environment}

The physical and psychosocial care environment was more of a barrier than a facilitator for a palliative approach in the ICU, and only two studies reported that support through a nurse-adapted bedside environment 


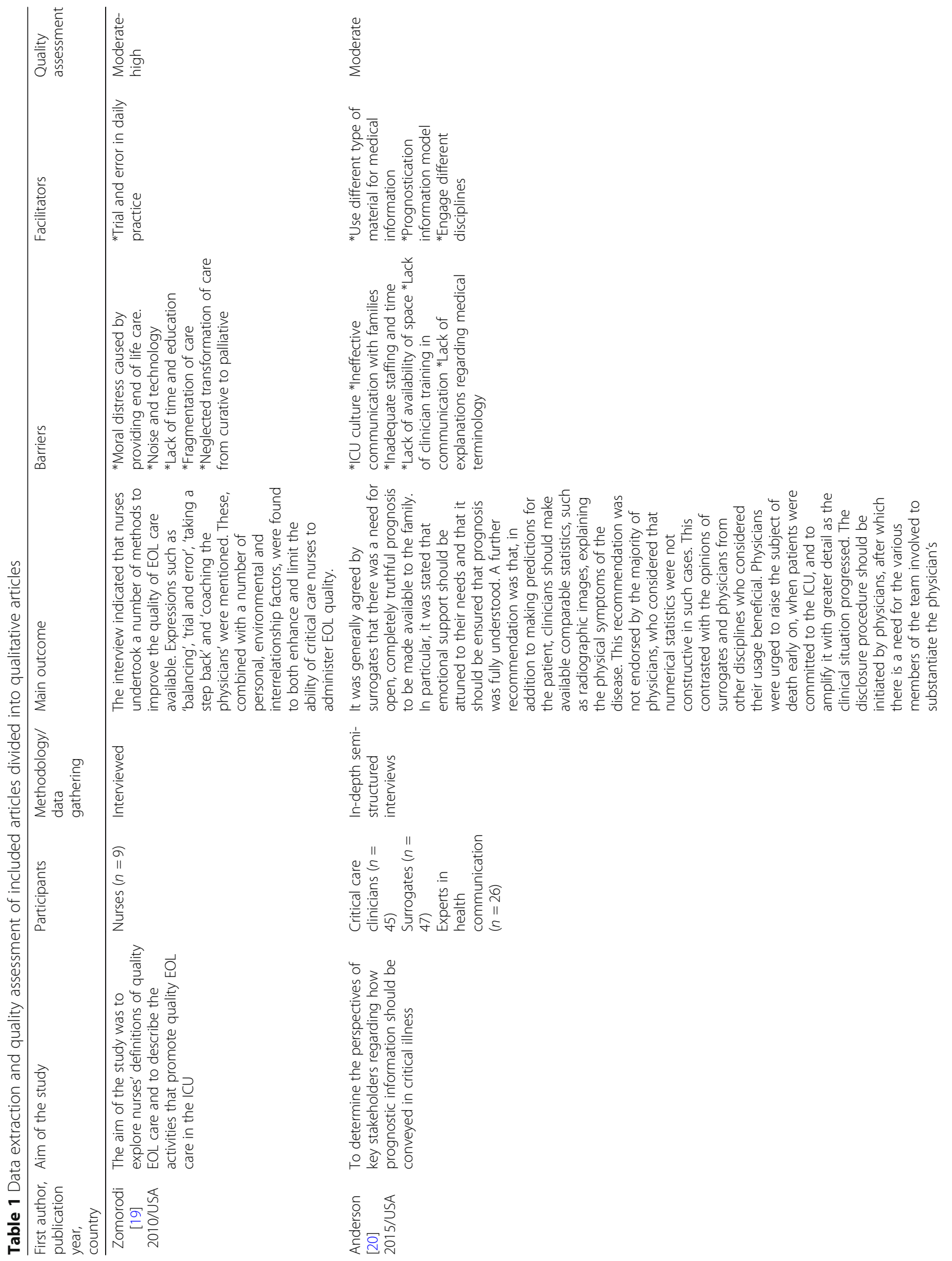




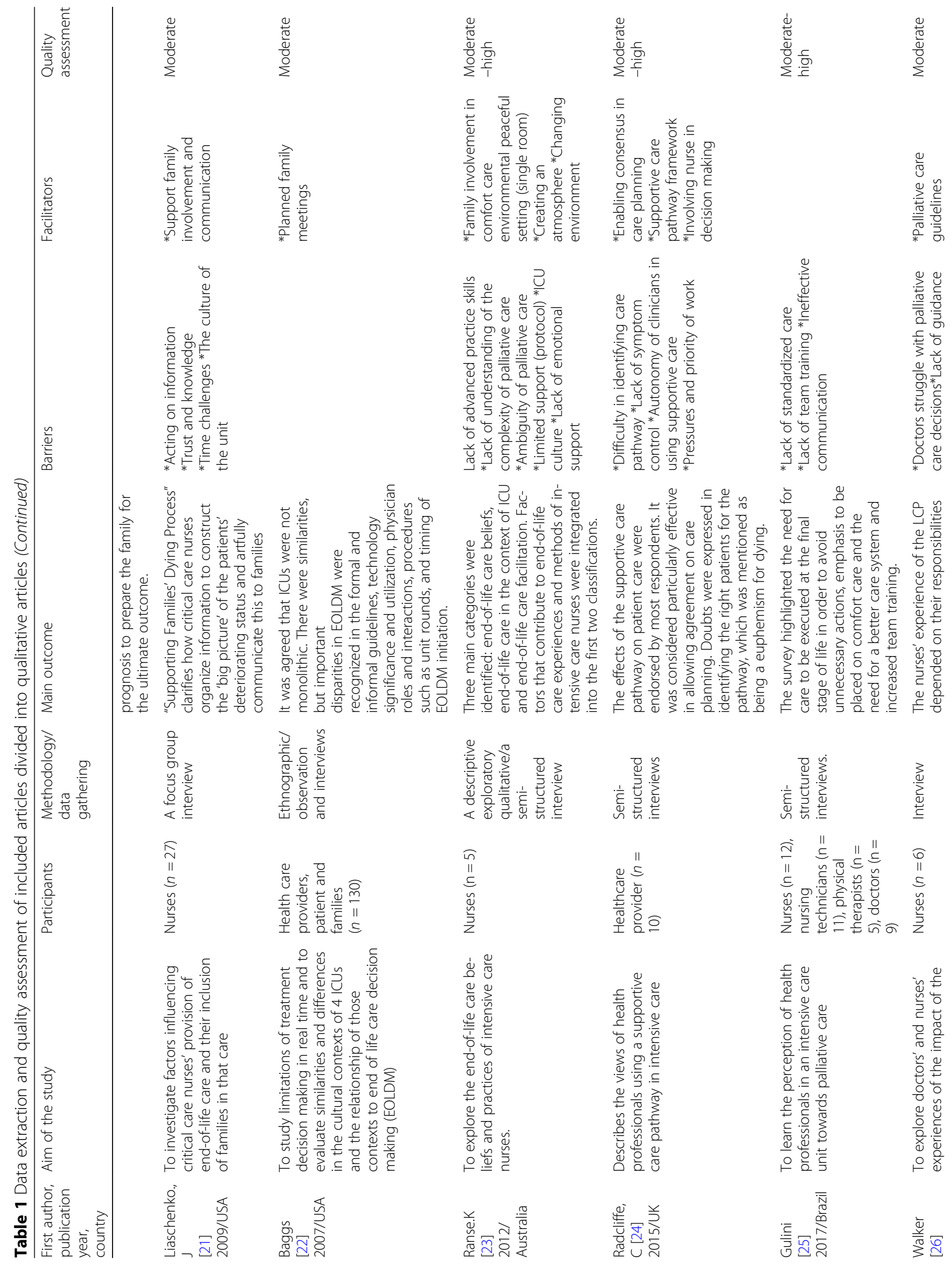




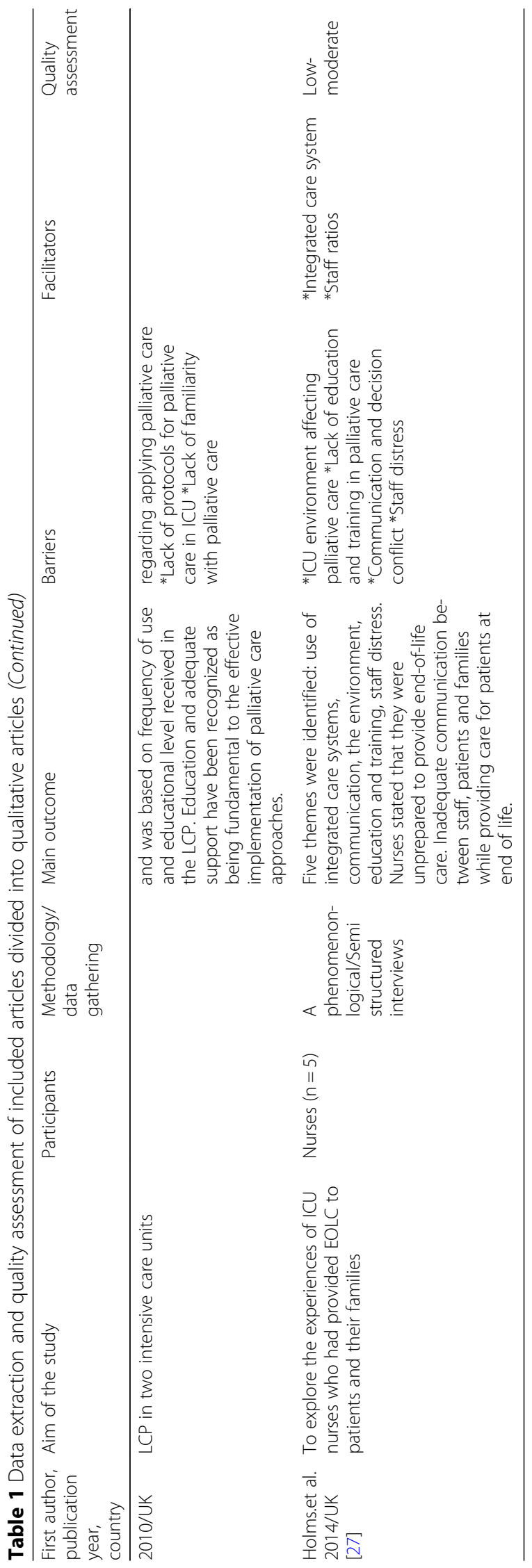




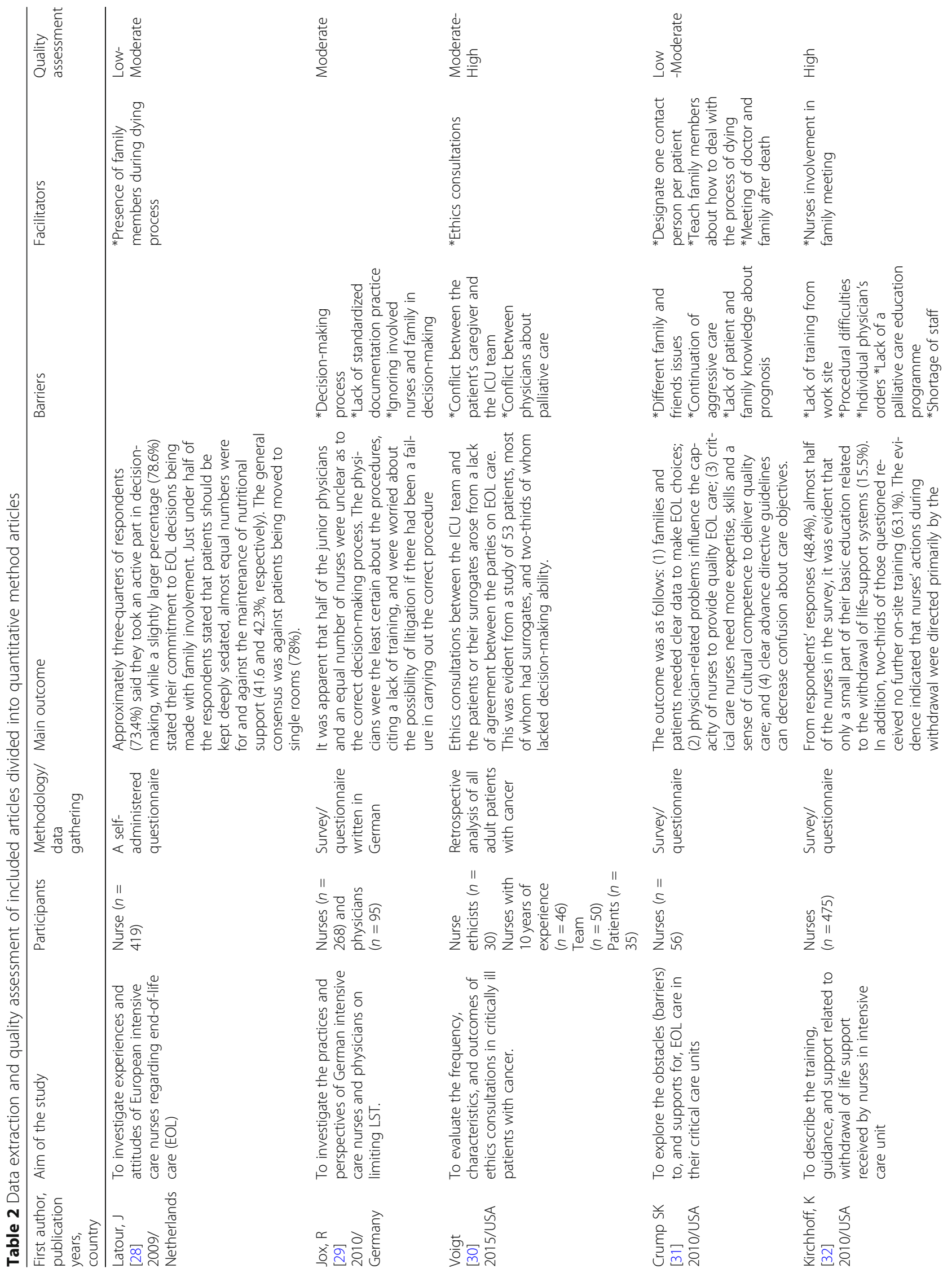




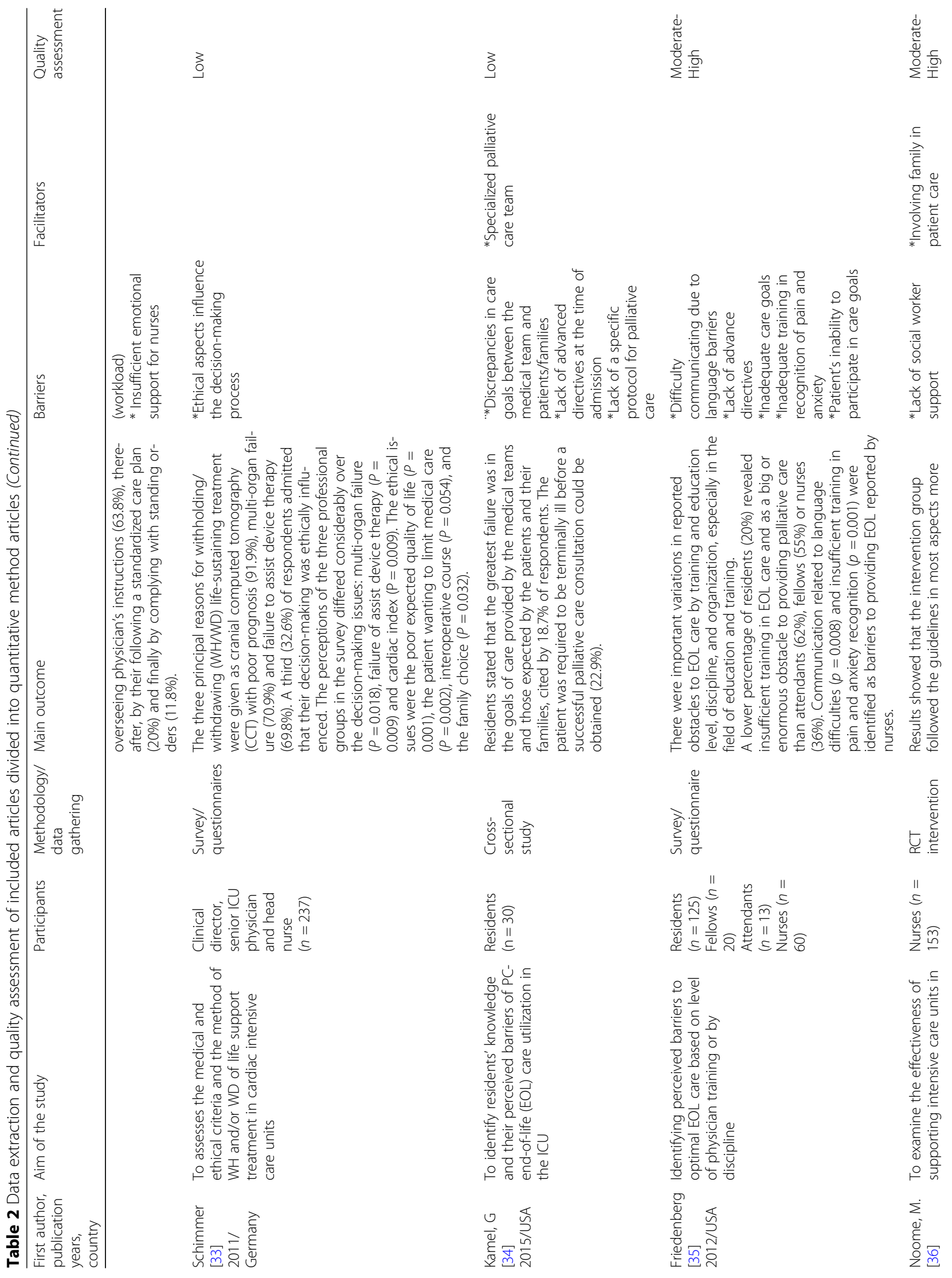




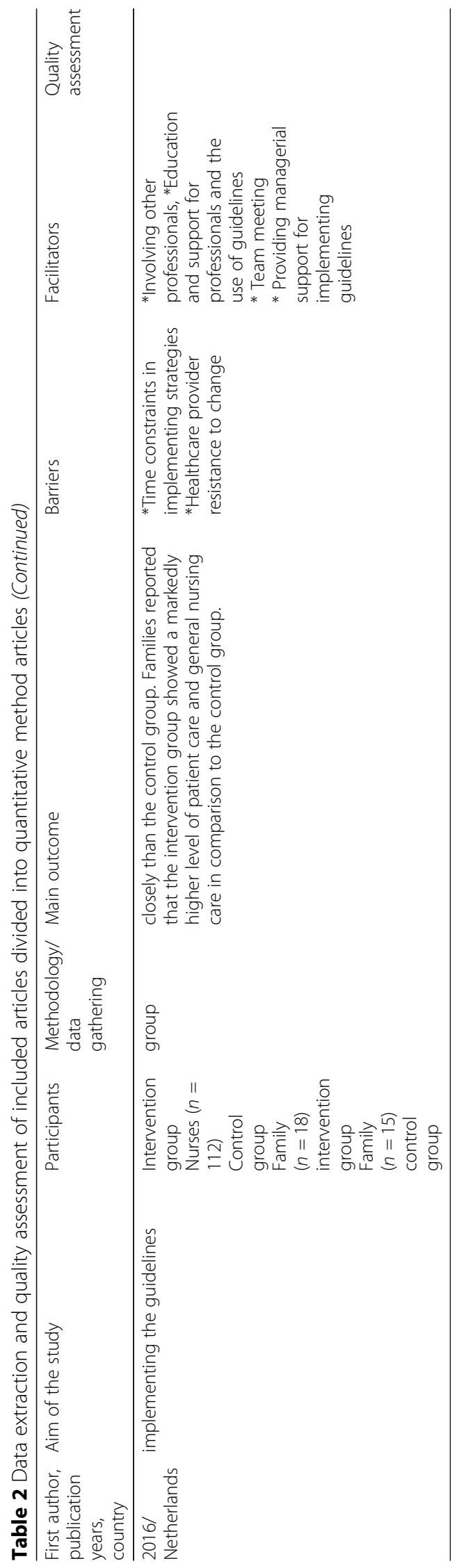




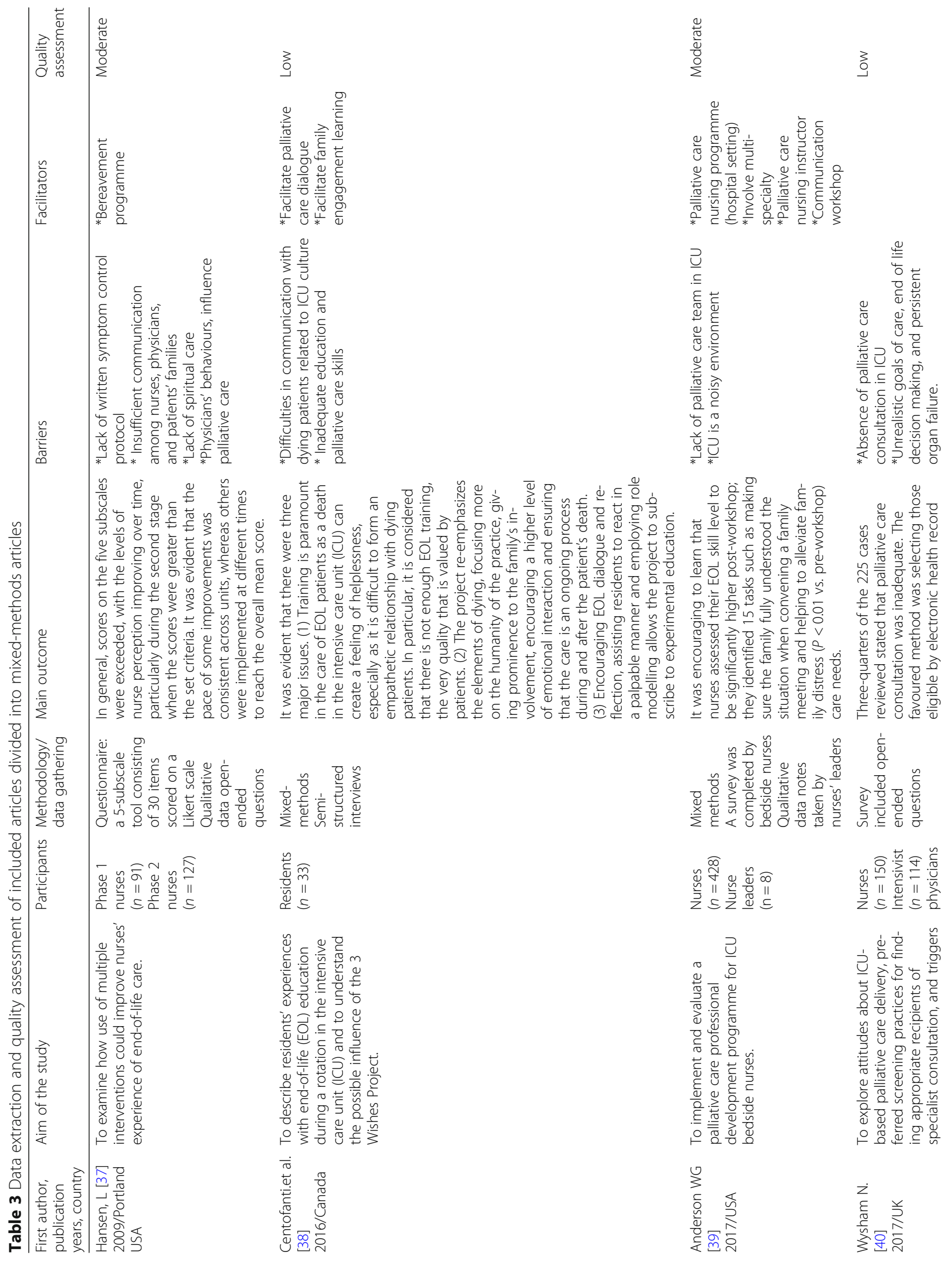




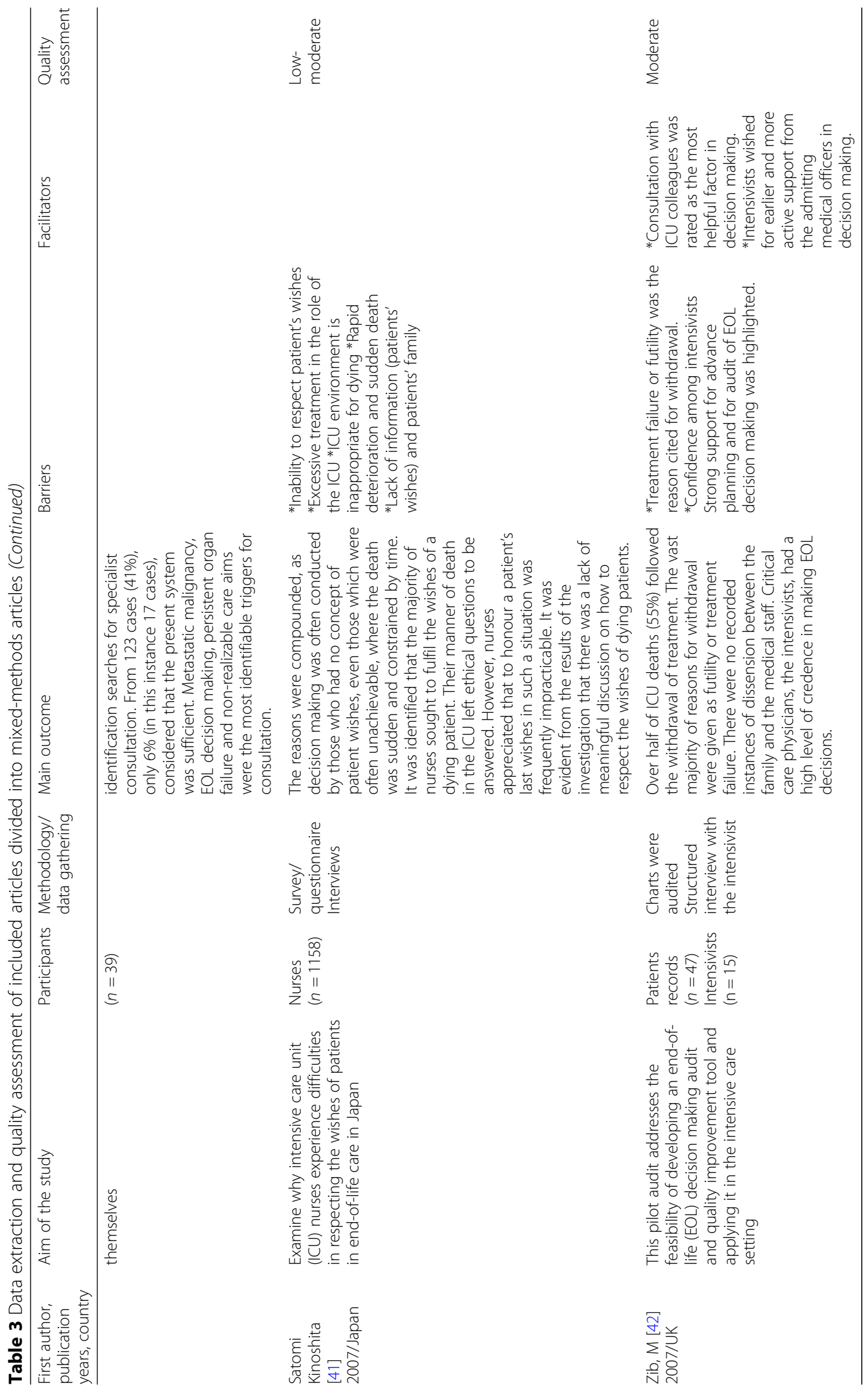




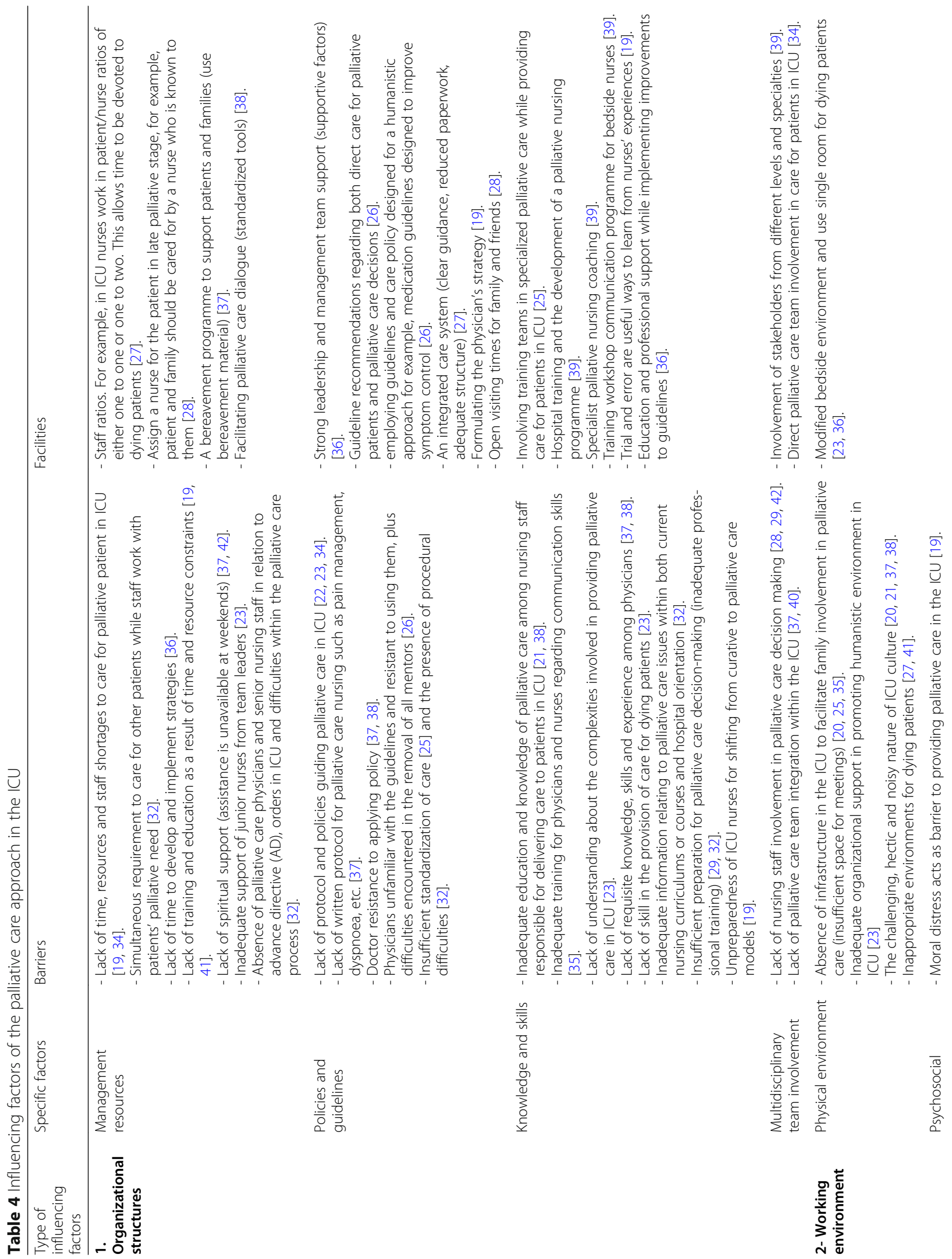




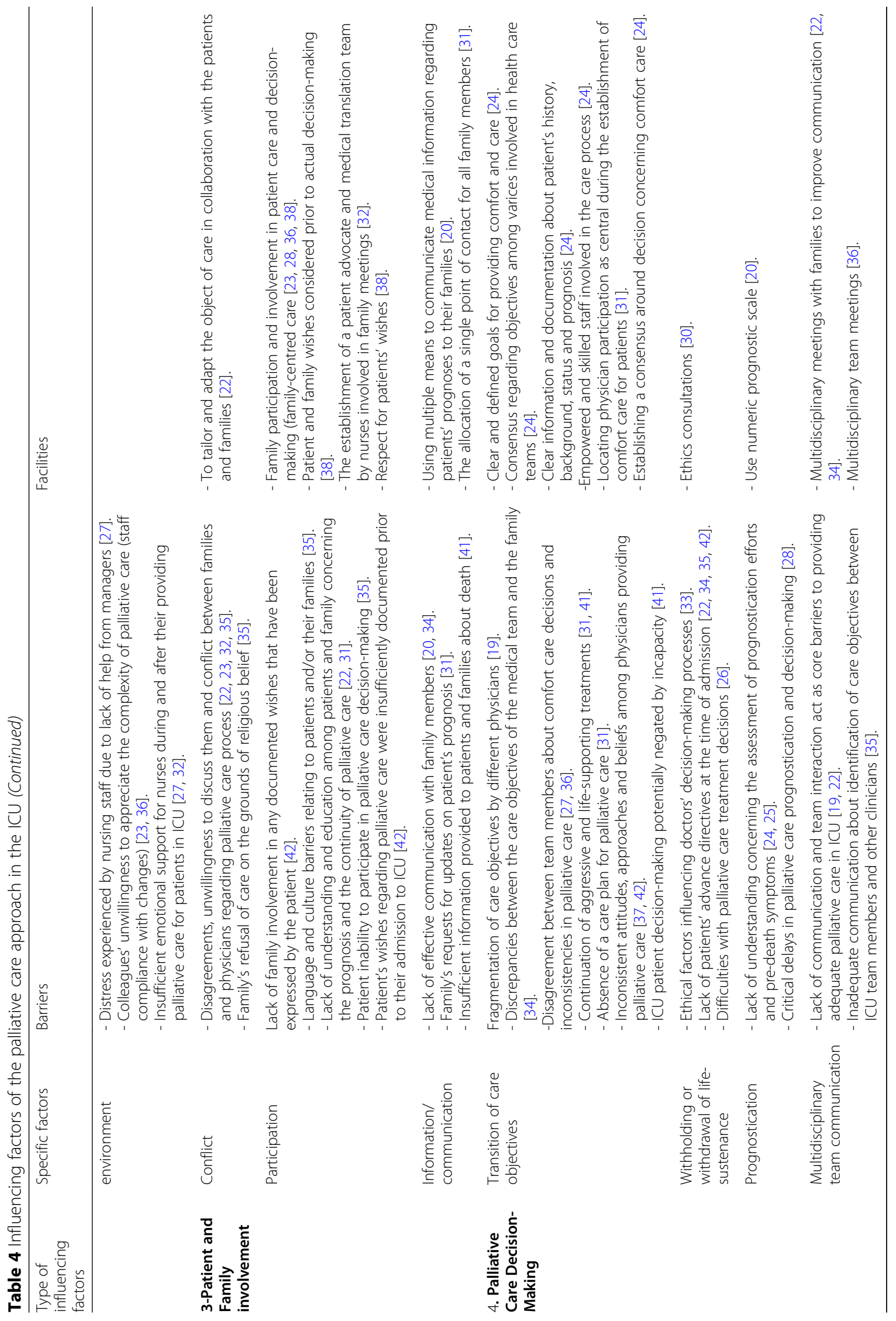


was effective in the ICU [36]. In many studies, the physical environment or infrastructure of the ward did not facilitate the support and participation of families while integrating a palliative approach for their patients [25, 35]. Identified barriers included a noisy environment with lack of privacy and confusion regarding who to approach for information [20,37, 38]. Increased moral distress and the need for emotional support to reduce such stress during the integration of a palliative approach in the ICU was described in two studies $[19,27]$, indicating a lack of support from managers, other staff and external support services $[27,32]$.

\section{Patient and family involvement}

Three studies emphasized that conflict and disagreements between family members and physicians concerning the goals of care were a barrier to the integration of a palliative approach [22, 32, 35], and other studies highlighted communication challenges with family members [20,34] related to language and culture [35], religious beliefs [35], and inadequate information about prognosis [31, 41]. Nevertheless, family involvement in patient care regarding the sharing of information, respect for others' wishes, and cooperation among patients, families and healthcare providers before they decided to change the goals of care towards a palliative orientation was found to be a facilitating factor $[28,36,38]$.

\section{Palliative care decision-making}

Studies report that continued intensive care intervention for patients was a barrier to making decisions about the integration of a palliative approach in the goals of care $[31,41]$, as well as a lack of understanding about how to assess patients' prognostication towards palliative care for dying patients and their family [24, 25]. For example, one study found that physicians were unable to identify patients who required a palliative approach to care in the early stage of intensive care [28]. Three studies emphasized that nurses did not contribute to the decision to integrate a palliative approach for their patients in the ICU $[28,29,42]$. Other identified barriers to decision making were physicians' attitudes and beliefs about palliative care [37, 42], disagreement between physicians [19] and the ICU team regarding the goals of care [27, 36], a lack of standardized care [25], and insufficient communication among team members $[19,22,35]$, all of which hampered integration in terms of transitions from lifesustaining interventions to palliative goals (see Table 4).

Clarity, agreement and documentation of the palliative goals of care decisions were identified as facilitators [24], together with ethical consultation [30,33] and the use of a numeric prognostic scale to support improved prognostication efforts [20].

\section{Discussion}

In this study, we sought to identify barriers to and facilitators of the integration of a palliative approach in intensive care units. To our knowledge, this study is the first systematic review that combines results based on qualitative and quantitative data to illuminate factors influencing a palliative approach in the critical care environment. Our results suggest that the transition from life-sustaining interventions to palliative goals of care in an intensive care context is hindered by both organizational and structural factors (e.g., resources, time constraints, workloads, and work environments) as well as individual factors (e.g., healthcare provider, patient, and family attitudes, communication, interaction and knowledge backgrounds). Our quality assessment suggests that the majority of articles $(n=16)$ were assessed to be of either moderate or moderate-to-high quality. The results from the four articles assessed to have low quality were supported by similar results from other studies included.

Today, a palliative approach to care is characterized by early identification of palliative care needs, adaptation of palliative care knowledge and integration into practice [43]. Given the complex nature of the intensive care context, such knowledge translation may become more challenging. There is no doubt that there is a need for the successful knowledge translation of a palliative approach into the ICU; however, the studies reviewed did not explicitly evaluate this. Through acknowledgment of the complexities involved, we need explicit knowledge translation research demonstrating valid implementation strategies. One way of moving towards knowledge translation is using the PARiHS (Promoting Action on Research Implementation in Health Services) model, which provides important insights for supporting knowledge translation into practice by focusing the implementation process on evidence, context and facilitation. According to the PARiHS model, the context/setting, as well as practice facilitators for change, are as important as the evidence supporting the knowledge. We use this model to discuss the findings from this study.

Organizational structures appeared in this study to be one of the barriers to the achievement of a palliative approach in the ICU. According to the PARiHS model, the context/setting, as well as practice facilitators for change, are as important as the evidence supporting the knowledge. The PARiHS framework demonstrates the need to address leadership and organizational aspects by understanding human relationships [44]. The present study shows that the lack of clinical guidelines and policies for integrating palliative care hinders implementation. Nevertheless, there is also evidence that professionals tend to disregard or be unaware of guidelines for a palliative approach in the ICU, pointing towards the importance of understanding professional perceptions and 
attitudes towards a palliative approach. This finding is in line with a review by Kahveci [14] that showed the impact of sociocultural factors and the lack of awareness of a palliative approach. Professionals' perceptions and attitudes, leadership and organizational aspects, as well as patients' and relatives' preferences and participation, need to be explored for successful integration. Thus, integration is both an organizational challenge as well as an individual challenge, as the organizational culture is created, sustained or changed by the people who work within the organization [45].

An important aspect of knowledge translation highlighted in the PARiHS framework is the context of care and its environment. Our results found more research emphasizing the challenges that the ICU physical work environment imposes on a palliative approach to care [30, 33, 42]. Interestingly, we found no articles pinpointing facilitating factors for the psychosocial care environment in the ICU. Therefore, future research should further investigate the care environment in various situations, such as how to support staff and reduce the stress of the care environment in general in this setting.

The present study highlights the importance of both patient and family involvement. The importance of family involvement is in line with the findings of previous literature $[8,9]$. Ineffective communication, a lack of family education, and a lack of healthcare provider awareness were shown to be key issues underlying conflict between family and physicians. Patient and family involvement are linked to knowledge and education [22,31], so it is vital to address family education programmes in the ICU. Studies show a palliative approach integrated into reduced patient and family distress [10-13]. This suggests a need for improved palliative care education and training to assess patients' and families' needs, wishes, and participation in care and goal setting in terms of a palliative orientation.

In the present study, decision-making to integrate a palliative approach in intensive care is influenced by healthcare professionals' knowledge and attitudes about the transition from curative-focused to palliative-focused goals of care, which highlights the importance of focusing on healthcare professionals' goal-setting attitudes and abilities in the integration of a palliative approach [46]. Unsurprisingly, having a clear goal of care on admission to the ICU seems to support professionals in the palliative decisionmaking process [24]. However, this cannot be considered in isolation, as many complex related factors can affect it, for example, the wishes of the patient and family and difficulties in defining a patient's prognosis on admission [35]. Studies regarding nurses' involvement in team decisionmaking or consulting the specialized palliative care team were scarce for palliative care in the ICUs $[28,29,42]$. Further studies may explore the impact of nurses' involvement in the decision-making process.

\section{Limitations of the study}

In this systematic literature review, we described factors influencing the integration of a palliative approach within the ICU. The majority of the included studies were assessed to be of moderate or moderate-to-high quality, with only one of these assessed to be of high quality, and four studies were assessed to be of low quality. The reader should thus acknowledge the heterogeneity of the study designs, as well as the spectrum of quality within the included studies. Although the heterogeneity of studies within a mixed-methods review could be acknowledged as a limitation, it is also a strength, as it provides a broad overview of the topic. As in many systematic studies, researchers' language skills are a limitation because we only included literature in English.

\section{Conclusion}

Factors hindering the integration of a palliative approach in an intensive care context are constituting a complex interplay among the organizational structure, the care environment and the clinician's perception and attitudes. While patient and family involvement were identified as an important facilitator of palliative care, it was also identified as a barrier for the clinicians due to challenges in shared goal setting and communication. We suggest that future integration efforts targeting a palliative approach should focus on organizational and educational efforts that strengthen human relationships and partnerships, not in the least regarding patient and family involvement. Moreover, there is a need for research evaluating useful strategies for the knowledge translation of a palliative approach in the ICU.

\section{Supplementary information}

Supplementary information accompanies this paper at https://doi.org/10. 1186/s12904-020-00616-y.

Additional file 1. All databases searched form 2017-12-22 to 2018-01-31. Additional file 2. Quality assessment A1-A4.

\section{Abbreviations}

ICU: Intensive care units; RCT: Randomized control trial; CASP: Critical Assessment Skills Program; MMAT: The Mixed Methods Appraisal Tool; Best BETs: Best Evidence topics; PARiHS: Promoting Action on Research Implementation in Health Services; PICOC: Population, Intervention, Comparison, Outcome and Context

\section{Acknowledgements}

We would like to acknowledge medical librarians Linda Hammarbäck and Magnus Holmberg, whose expertise was helpful in formulating and finalizing the search terms for this review.

\section{Authors' contributions}

All authors contributed to the design, conception, and development of the study. $\mathrm{HH}$ and $\mathrm{AW}$ screened and reviewed all articles. $\mathrm{HH}$ drafted the manuscript. HH, AW, SO, and JÖ discussed, revised and confirmed the findings. All authors were responsible for the critical revision and finalization of the manuscript. All authors read and approved the final manuscript. 


\section{Funding}

Open access funding provided by University of Gothenburg. HH's PhD scholarship is funded by Princess Nourah bint Abdulrahman University, Riyadh, Saudi Arabia; however, the funding body did not have any influence in the design of the study; the collection, analysis, interpretation of the data; or the writing of the manuscript.

\section{Availability of data and materials}

Data sharing is not applicable to this article, as no datasets were generated or analysed during the current study.

\section{Ethics approval and consent to participate}

Not applicable.

\section{Consent for publication}

Not applicable, as it is a systematic review.

\section{Competing interests}

The authors declare that they have no competing interests.

\section{Author details}

Institute of Health and Care Sciences, Sahlgrenska Academy, University of Gothenburg, Gothenburg, Sweden. ${ }^{2}$ Princess Nourah bint Abdulrahman University, Riyadh, Saudi Arabia. ${ }^{3}$ Institute of Health and Care Sciences and University of Gothenburg Centre for Person-Centred Care, Sahlgrenska Academy at the University of Gothenburg, Gothenburg, Sweden. ${ }^{4}$ Palliative Centre, Sahlgrenska University Hospital Region Västra Götaland, Gothenburg, Sweden. ${ }^{5}$ Department of Anaesthesiology and Intensive Care Medicine, Region Västra Götaland, Sahlgrenska University Hospital/Östra, Gothenburg, Sweden

Received: 26 September 2019 Accepted: 7 July 2020

Published online: 22 July 2020

\section{References}

1. Lanken PN, Manaker S, Kohl BA, Hanson III CW: Intensive care unit manual Ebook. 2nd ed. Elsevier Health Sciences; 2013.

2. Hardin KA, Meyers F, Louie S: Integrating palliative care in severe chronic obstructive lung disease. Journal of Chronic Obstructive Pulmonary Disease. 2008;5(4):207-20

3. Meier DE, Brawley OW. Palliative care and the quality of life. J Clin Oncol. 2011;29(20):2750-2.

4. Lueck C, Stadler M, Koenecke C, Hoeper MM, Dammann E, Schneider A, Kielstein JT, Ganser A, Eder M, Beutel G. Improved short- and long-term outcome of allogeneic stem cell recipients admitted to the intensive care unit: a retrospective longitudinal analysis of 942 patients. Intensive Care Med. 2018;44(9):1483-92.

5. Narayan M, Kashuk JL: Admission/Discharge Criterion for Acute Care Surgery Patients in the ICU: A General Review of ICU Admission and Discharge Indications. In: Intensive Care for Emergency Surgeons. edn. Edited by Picetti E, Pereira BM, Razek T, Narayan M, Kashuk JL. Cham: Springer International Publishing; 2019: 1-21.

6. Rosenbaum, L. Facing Covid-19 in Italy-ethics, logistics, and therapeutics on the epidemic's front line. N Engl J Med. 2020;382(20):1873-75.

7. Truog RD, Mitchell C, Daley GQ. The toughest triage-allocating ventilators in a pandemic. N Engl J Med. 2020;382(21):1973-75.

8. Meier DE. Increased access to palliative care and hospice services: opportunities to improve value in health care. Milbank Quarterly. 2011;89(3): 343-80.

9. Schulz V, Novick RJ: The distinct role of palliative care in the surgical intensive care unit. In: Seminars in cardiothoracic and vascular anesthesia: 2013: SAGE publications Sage CA: Los Angeles; 2013: 240-248.

10. Adolph MD, Frier KA, Stawicki SPA, Gerlach AT, Papadimos TJ. Palliative critical care in the intensive care unit: a 2011 perspective. Int J Critical Illness Injury Sci. 2011;1(2):147-53.

11. Aslakson R, Cheng J, Vollenweider D, Galusca D, Smith TJ, Pronovost PJ. Evidence-based palliative Care in the Intensive Care Unit: a systematic review of interventions. J Palliat Med. 2014:17(2):219-35.

12. Maddocks M, Lovell N, Booth S, Man WDC, Higginson IJ. Palliative care and management of troublesome symptoms for people with chronic obstructive pulmonary disease. Lancet. 2017;390(10098):988-1002.
13. Nelson JE, Puntillo KA, Pronovost PJ, Walker AS, MCAdam JL, llaoa D, Penrod J. In their own words: patients and families define high-quality palliative care in the intensive care unit. Crit Care Med. 2010;38(3):808-18.

14. Kahveci K. Palliative care and intensive care integration. Med Sci Int Med J. 2017;6(3):603-9.

15. Hawley P. Barriers to access to palliative care. Palliat Care. 2017:10: 1178224216688887

16. Dumanovsky T, Augustin R, Rogers M, Lettang K, Meier DE, Morrison RS. The growth of palliative Care in U.S. hospitals: a status report. J Palliat Med. 2016;19(1):8-15.

17. Booth A, Sutton A, Papaioannou D: Systematic approaches to a successful literature review: Sage; 2016.

18. Thomas J, Harden A. Methods for the thematic synthesis of qualitative research in systematic reviews. BMC Med Res Methodol. 2008;8(1):1.

19. Zomorodi M, Lynn MR. Critical care nurses' values and behaviors with endof-life care: perceptions and challenges. J Hosp Palliat Nurs. 2010;12(2):8998.

20. Anderson WG, Cimino JW, Ernecoff NC, Ungar A, Shotsberger KJ, Pollice LA, Buddadhumaruk P, Carson SS, Curtis JR, Hough CL. A multicenter study of key stakeholders' perspectives on communicating with surrogates about prognosis in intensive care units. Ann Am Thoracic Society. 2015;12(2):14252.

21. Liaschenko J, O'Conner-Von S, Peden-McAlpine C. The" big picture": communicating with families about end-of-life care in intensive care unit. Dimensions Critical Care Nurs. 2009;28(5):224-31.

22. Baggs JG, Norton SA, Schmitt MH, Dombeck MT, Sellers CR, Quinn JR. Intensive care unit cultures and end-of-life decision making. J Crit Care. 2007;22(2):159-68.

23. Ranse $K$, Yates $P$, Coyer F. End-of-life care in the intensive care setting: a descriptive exploratory qualitative study of nurses' beliefs and practices. Aust Crit Care. 2012;25(1):4-12.

24. Radcliffe C, Hewison A. Use of a supportive care pathway for end-of-life care in an intensive care unit: a qualitative study. Int J Palliat Nurs. 2015;21(12): $608-15$.

25. Gulini J, do Nascimento ERP, Moritz RD, da Rosa LM, Silveira NR, Vargas MAD: Intensive Care Unit team perception of palliative care: the discourse of the collective subject. Revista Da Escola De Enfermagem Da Usp 2017, 51:6.

26. Walker R, Read S. The Liverpool care pathway in intensive care: an exploratory study of doctor and nurse perceptions. Int J Palliat Nurs. 2010; 16(6):267-73.

27. Holms N, Milligan S, Kydd A. A study of the lived experiences of registered nurses who have provided end-of-life care within an intensive care unit. Int J Palliat Nurs. 2014;20(11):549-56.

28. Latour JM, Fulbrook P, Albarran JW. EfCCNa survey: European intensive care nurses' attitudes and beliefs towards end-of-life care. Nursing Critical Care. 2009:14(3):110-21.

29. Jox RJ, Krebs M, Fegg M, Reiter-Theil S, Frey L, Eisenmenger W, Borasio GD. Limiting life-sustaining treatment in German intensive care units: a multiprofessional survey. J Crit Care. 2010;25(3):413-9.

30. Voigt LP, Rajendram P, Shuman AG, Kamat S, McCabe MS, Kostelecky N, Pastores SM, Halpern NA. Characteristics and outcomes of ethics consultations in an oncologic intensive care unit. J Intensive Care Med. 2015;30(7):436-42

31. Crump SK, Schaffer MA, Schulte E. Critical care nurses' perceptions of obstacles, supports, and knowledge needed in providing quality end-of-life care. Dimensions Critical Care Nurs. 2010;29(6):297-306.

32. Kirchhoff KT, Kowalkowski JA. Current practices for withdrawal of life support in intensive care units. Am J Crit Care. 2010;19(6):532-41.

33. Schimmer C, Gorski A, Özkur M, Sommer SP, Hamouda K, Hain J, Aleksic I, Leyh R. Policies of withholding and withdrawal of life-sustaining treatment in critically ill patients on cardiac intensive care units in Germany: a national survey. Interact Cardiovasc Thorac Surg. 2012;14(3):294-9.

34. Kamel G, Paniagua M, Uppalapati A. Palliative Care in the Intensive Care Unit: are residents well trained to provide optimal care to critically ill patients? Am J Hosp Palliat Care. 2015;32(7):758-62.

35. Friedenberg AS, Levy MM, Ross S, Evans LE. Barriers to end-of-life Care in the Intensive Care Unit: perceptions vary by level of training, discipline, and institution. J Palliat Med. 2012;15(4):404-11.

36. Noome M, Dijkstra BM, Leeuwen E, Vloet LCM. Effectiveness of supporting intensive care units on implementing the guideline 'End-of-life care in the 
intensive care unit, nursing care': a cluster randomized controlled trial. J Adv Nurs. 2017;73(6):1339-54.

37. Hansen L, Goodell TT, Dehaven J, Smith M. Nurses' perceptions of end-oflife care after multiple interventions for improvement. Am J Crit Care. 2009; 18(3):263-71.

38. Centofanti J, Swinton M, Dionne J, Barefah A, Boyle A, Woods A, Shears M, Heels-Ansdell D, Cook D. Resident reflections on end-of-life education: a mixed-methods study of the 3 wishes project. BMJ Open. 2016;6(3):e010626.

39. Anderson WG, Puntillo K, Cimino J, Noort J, Pearson D, Boyle D, Grywalski M, Meyer J, O'Neil-Page E, Cain J. Palliative care professional development for critical care nurses: a multicenter program. Am J Crit Care. 2017;26(5): 361-71.

40. Wysham NG, Hua M, Hough CL, Gundel S, Docherty SL, Jones DM, Reagan O, Goucher H, McFarlin J, Cox CE. Improving ICU-based palliative care delivery: a multicenter, multidisciplinary survey of critical care clinician attitudes and beliefs. Crit Care Med. 2017;45(4):e372-8.

41. Kinoshita S. Respecting the wishes of patients in intensive care units. Nurs Ethics. 2007;14(5):651-64

42. Zib M, Saul P. A pilot audit of the process of end-of-life decision-making in the intensive care unit. Crit Care Resusc. 2007;9(2):213-8.

43. Sawatzky R, Porterfield P, Lee J, Dixon D, Lounsbury K, Pesut B, Roberts D, Tayler C, Voth J, Stajduhar K. Conceptual foundations of a palliative approach: a knowledge synthesis. BMC Palliative Care. 2016;15(1):5.

44. Kitson A, Harvey G, McCormack B. Enabling the implementation of evidence based practice: a conceptual framework. Quality Health Care. 1998;7(3):14958.

45. Wolf A, Ekman I, Dellenborg L. Everyday practices at the medical ward: a 16month ethnographic field study. BMC Health Serv Res. 2012;12(1):184.

46. Weiss M, Michalsen A, Toenjes A, Porzsolt F, Bein T, Theisen M, Brinkmann A, Groesdonk H, Putensen C, Bach F, et al. End-of-life perceptions among physicians in intensive care units managed by anesthesiologists in Germany: a survey about structure, current implementation and deficits. BMC Anesthesiol. 2017:17(1):93.

\section{Publisher's Note}

Springer Nature remains neutral with regard to jurisdictional claims in published maps and institutional affiliations.

Ready to submit your research? Choose BMC and benefit from:

- fast, convenient online submission

- thorough peer review by experienced researchers in your field

- rapid publication on acceptance

- support for research data, including large and complex data types

- gold Open Access which fosters wider collaboration and increased citations

- maximum visibility for your research: over $100 \mathrm{M}$ website views per year

At $\mathrm{BMC}$, research is always in progress.

Learn more biomedcentral.com/submissions 